\title{
Os impactos psicológicos de pacientes pós-bariátricas: uma revisãode literatura
}

\author{
The psychological impacts of post-bariatric patients: a literature review \\ Los impactos psicológicos de los pacientes posbariátricos: una revisión de la literatura
}

Recebido: 29/11/2021 | Revisado: 02/12/2021 | Aceito: 13/12/2021 | Publicado: 21/12/2021

\author{
Bruna Marcy Portela da Costa \\ ORCID: https://orcid.org/0000-0002-8020-1413 \\ Faculdade de Ensino Superior do Piauí, Brasil \\ E-mail: brunamarcy12@gmail.com \\ Ruth Raquel Soares de Farias \\ ORCID: https://orcid.org/0000-0002-0988-0900 \\ Faculdade de Ensino Superior do Piauí, Brasil \\ E-mail: ruthraquelsf@gmail.com \\ Sara Cavalcanti Souza \\ ORCID: https://orcid.org/0000-0003-3795-7431 \\ Centro de Ensino Unificado do Piauí, Brasil \\ E-mail: scavalcantis@yahoo.com.br \\ Gislene Mariana Pereira Castelo Branco \\ ORCID: https://orcid.org/0000-0003-4393-771X \\ Faculdade de Ensino Superior do Piauí, Brasil \\ E-mail: gi.marianna@hotmail.com
}

\begin{abstract}
Resumo
A obesidade é uma doença crônica grave que apresenta como principal característica o acúmulo exagerado de gordura corporal que, segundo a OMS é responsável pelo comprometimento da saúde de aproximadamente 4 milhões de indivíduos. Os objetivos do estudo são analisar os aspectos psicológicos em pacientes submetidos a cirurgia bariátrica, além de relacionar o comportamento de adesão de pacientes pós-bariátricas com as indicações da equipe multiprofissional e assim categorizar os possíveis fatores que ocasionam o reganho de peso dos pacientes pósbariátricos. Essa é uma pesquisa do tipo revisão integrativa da literatura, desenvolvida com a coleta de artigos nas bases de dados online: Literatura Científica e Técnica da América Latina e Caribe (LILACS), Sistema Online de Busca e Análise de Literatura Médica (MEDLINE) e na base eletrônica Scientific Eletronic Library Online (SCIELO), por meio dos descritores: "Cirurgia Bariátrica" (Bariatric Surgery) e "Saúde Mental” (Mental Health) e "transtornos pós-bariátrica" (Post-bariatric Disorders), associados ao operador booleano AND. Os dados foram coletados em agosto de 2021. Foram analisados na pesquisa 123 artigos originais disponíveis na íntegra, no idioma português e inglês compreendido entre os anos de 2016 a 2021. E foram excluídos 106 artigos por não estarem dentro do corte temporal de publicação de até 5 anos ou que não estava no foco desta revisão, e os que não contemplavam os critérios de inclusão. A amostra final ficou com 15 publicações. Os resultados demonstraram que a ansiedade e o medo são os aspectos pisocológicos mais relatados e o acompanhamento multiprofissional regular pós-cirurgia bariátrica está associado a melhores resultados cirúrgicos.
\end{abstract}

Palavras-chave: Cirurgia bariátrica; Psicologia; Saúde mental; Transtornos pós-bariátrica.

\begin{abstract}
Obesity is a serious chronic disease whose main characteristic is the exaggerated accumulation of body fat which, according to the WHO, is responsible for the health impairment of approximately 4 million individuals. The objectives of the study are to analyze the psychological aspects of patients undergoing bariatric surgery, in addition to relating the adherence behavior of post-bariatric patients to the indications of the multidisciplinary team and thus categorize the possible factors that cause weight regain in post-bariatric patients. bariatric. This is an integrative literature review type research, developed with the collection of articles in online databases: Scientific and Technical Literature of Latin America and the Caribbean (LILACS), Online System for Search and Analysis of Medical Literature (MEDLINE) and in electronic database Scientific Electronic Library Online (SCIELO), through the descriptors: "Bariatric Surgery" (Bariatric Surgery) and "Mental Health" (Mental Health) and "Post-bariatric disorders" (Post-bariatric Disorders), associated with the Boolean operator AND. . Data were collected in August 2021. 123 original articles available in full, in Portuguese and English from 2016 to 2021, were analyzed in the research. 106 articles were excluded for not being within the publication period of up to 5 years, that were beyond the focus of this review, and those that did not meet the inclusion criteria. The final sample consisted of 15 publications. The results showed that anxiety and fear are the most reported pisocological aspects and that regular multiprofessional follow-up after bariatric surgery is associated with better surgical results.
\end{abstract}

Keywords: Bariatric surgery; Psychology; Mental health; Post-bariatric disorders. 


\begin{abstract}
Resumen
La obesidad es una enfermedad crónica grave cuya principal característica es la acumulación exagerada de grasa corporal, que, según la OMS, es responsable del compromiso de salud de aproximadamente 4 millones de personas. Los objetivos del estudio son analizar los aspectos psicológicos de los pacientes sometidos a cirugía bariátrica, además de relacionar el comportamiento de adherencia de los pacientes posbariátricos con las indicaciones del equipo multidisciplinar y así categorizar los posibles factores que provocan la recuperación de peso en el posbariátrico. pacientes bariátricos. Se trata de una investigación tipo revisión integradora de la literatura, desarrollada con la recopilación de artículos en bases de datos en línea: Literatura Científica y Técnica de América Latina y el Caribe (LILACS), Sistema en Línea de Búsqueda y Análisis de Literatura Médica (MEDLINE) y en base de datos electrónica. Biblioteca electrónica en línea (SCIELO), a través de los descriptores: "Cirugía bariátrica" (Cirugía bariátrica) y "Salud mental" (Salud mental) y "Desórdenes posbariátricos" (Desórdenes posbariátricos), asociados al operador booleano AND. Los datos fueron recolectados en agosto de 2021. En la investigación se analizaron 123 artículos originales disponibles en su totalidad, en portugués e inglés de 2016 a 2021.5 años o que no fueron el foco de esta revisión, y aquellos que no cumplieron con la inclusión Criterios. La muestra final estuvo formada por 15 publicaciones. Los resultados mostraron que la ansiedad y el miedo son los aspectos pisocológicos más reportados y que el seguimiento multiprofesional regular después de la cirugía bariátrica se asocia con mejores resultados quirúrgicos.
\end{abstract}

Palabras clave: Cirugía bariátrica; Psicología; Salud mental; Trastornos posbariátricos.

\title{
1. Introdução
}

A obesidade é uma doença crônica com causas variadas, que contribui para sérios riscos na saúde, tanto a nível físico, quanto psíquico. Todavia, é primordial entender que a obesidade deve ser entendida como uma doença, deve-se entendê-la como um sintoma, que é o resultado de numerosos aspectos biopsicossociais (Macedo et al., 2019).

A doença funciona como fator de risco para várias doenças dentre as quais podem ser citados: dificuldades respiratórias, problemas dermatológicos e distúrbios do aparelho locomotor, além de favorecer o surgimento de enfermidades potencialmente letais como dislipidemias, doenças cardiovasculares, Diabetes Não-Insulino-Dependente (Diabetes Tipo II) e certos tipos de câncer. Contudo, o grau de excesso de gordura, sua distribuição corpórea e as consequências para a saúde apresentam variação entre os obesos (Moura et al., 2021).

Uma pesquisa divulgada pelo Instituto Brasileiro de Geografia e Estatística (IBGE) mostra que 95,9 milhões de brasileiros acima de 18 anos estão com excesso de peso, sendo que 41,2 milhões deles são considerados obesos. Cerca de 96 milhões de pessoas acima do peso representam 60,3\% dos brasileiros com mais de 18 anos, segundo os dados da Pesquisa Nacional de Saúde (PNS) (Brasil, 2020).

O aumento exagerado do peso corporal é uma tendência mundial exatamente por isso, muitas pessoas recorrem a cirurgia bariátrica para perder peso. Essa cirurgia é realizada com o objetivo de reduzir o peso de pessoas com o Índice de Massa Corporal (IMC) elevado, estimado pelo cálculo do peso corporal, em quilogramas, dividido pelo quadrado da altura $(\mathrm{IMC}=\mathrm{kg} / \mathrm{h} 2(\mathrm{~m}))$, e também pelo risco de mortalidade associada. É considerado obeso o indivíduo que se encontra acima de $30 \mathrm{~kg} / \mathrm{m}^{2}$ (Cassin et al., 2020).

A OMS define a gravidade da obesidade em: grau I (moderado excesso de peso), quando o IMC situa- se entre 30 e $34,9 \mathrm{~kg} / \mathrm{m}^{2}$; a obesidade grau II (obesidade leve ou moderada) com IMC entre 35 e 39,9 kg/m² e, por fim, obesidade grau III (obesidade mórbida) na qual IMC ultrapassa $40 \mathrm{~kg} / \mathrm{m}^{2}$ (Bardal et al., 2016).

Assim, o presente estudo tem por objetivos analisar os aspectos psicológicos em pacientes submetidos a cirurgia bariátrica, além de relacionar o comportamento de adesão de pacientes pós-bariátricos com as indicações da equipe multiprofissional e assim categorizar os possíveis fatores que ocasionam o reganho de peso dos pacientes pós-bariátricos. 


\section{Metodologia}

Trata-se de uma revisão integrativa da literatura, que possibilitou reunir as publicações sobre os impactos psicológicos em pacientes pós-bariátricos, a partir da análise dos objetivos para a obtenção de conclusões a partir da pergunta norteadora (Ercole et al., 2014).

A pesquisa procurou solucionar a seguinte questão: quais são os impactos psicológicos em pacientes pós-bariátricos? Para a construção de tal pesquisa foi utilizada o método de pesquisa PICO, em que P (população), pacientes pós-bariátrico; I (interesse), transtornos psicológicos; e o Co (contexto), Cirurgia Bariátrica.

O levantamento bibliográfico foi realizado por meio de indexadores presentes na Biblioteca Virtual em Saúde (BVS), tais como: Literatura Científica e Técnica da América Latina e Caribe (LILACS), Sistema Online de Busca e Análise de Literatura Médica (MEDLINE) e na base eletrônica Scientific Eletronic Library Online (SCIELO), por meio dos descritores: "Cirurgia Bariátrica” (Bariatric Surgery), "Saúde Mental” (Mental Health) e "transtornos pós-bariátrica” (Postbariatric Disorders), associados ao operador booleano AND.

Foram selecionados inicialmente na pesquisa 123 artigos originais disponíveis na íntegra, no idioma português e inglês compreendido entre os anos de 2016 a 2021. E foram excluídos 108 artigos por não contemplar o foco desta revisão, assim como os que estavam repetidos, e os que apresentavam textos incompletos. Após análise do título e resumo, e objetivo de estudo, selecionou-se 15 artigos para a amostra final.

Figura 1: Fluxograma do processo de busca e seleção dos artigos para a revisão integrativa de litertura. Teresina (PI), Brasil, 2021.

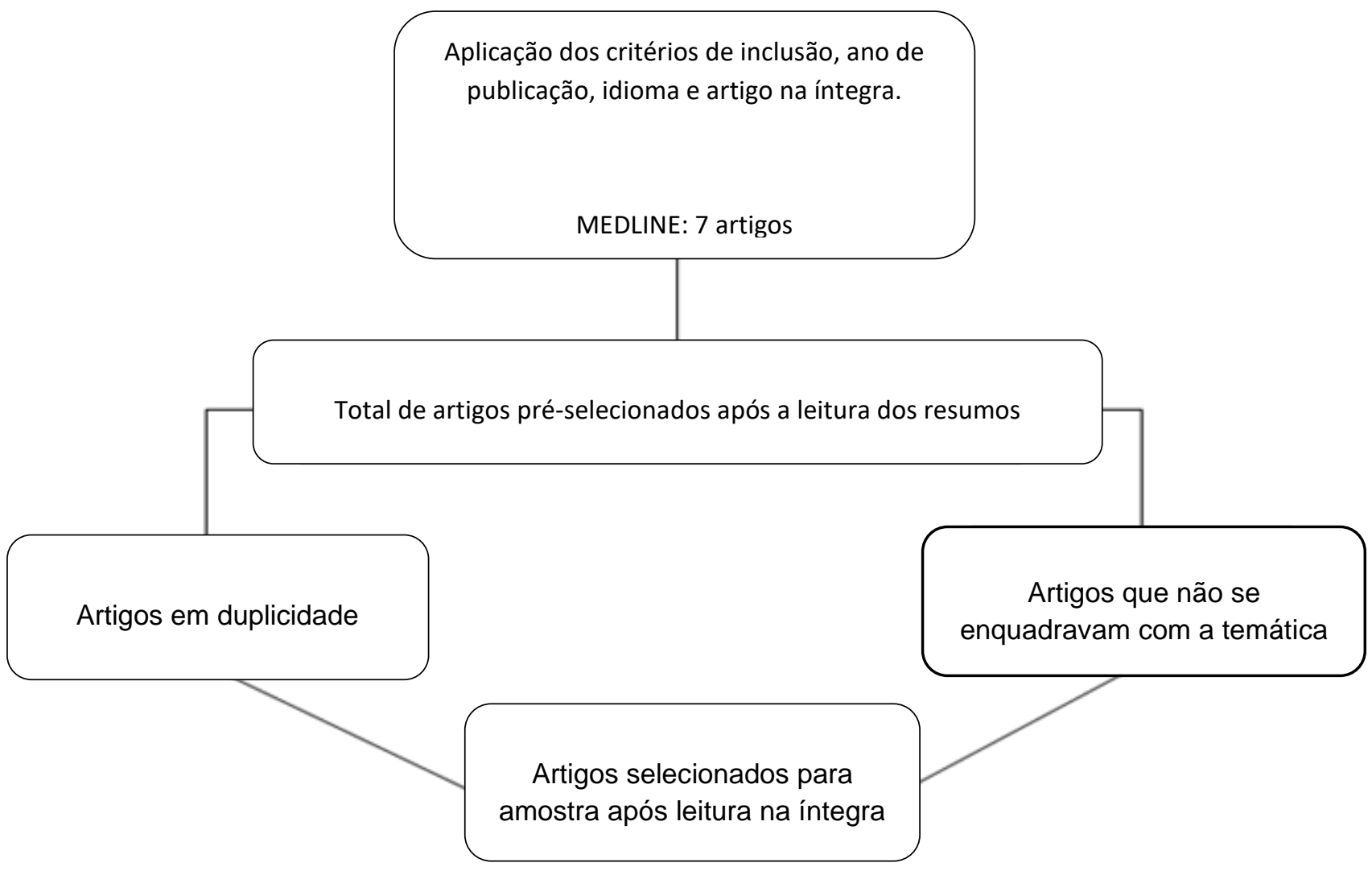

Fonte: Dados da pesquisa (2021). 
No processamento dos dados para a seleção dos artigos foram observadas algumas características dos estudos, por meio de um formulário adaptado do Ferreira et al. (2016) contendo identificação do artigo (autores, título, objetivos, metodologia e resultados).

A análise dos dados da pesquisa ocorreu em etapas significativas, a primeira foi a pré-análise que constituiu na escolha dos artigos a serem analisados; enfatizando as hipóteses e os objetivos incluídos inicialmente. O exame de todo o material buscou a compreensão da temática tradicional que realiza a primeira fase com recorte do texto, caracterizado na préanálise, com ação dos resultados obtidos com suas devidas interpretações que possibilitarão o esclarecimento das informações colhidas (Cerqueira et al. 2018).

Os resultados foram apresentados em tabelas que mostraram os elementos que construíram a ideia sobre o tema, ao passo que a discussão desses resultados ocorreu com base no referencial publicado e na correlação dos artigos selecionados apresentando os pontos principais e as possíveis diferenças entre eles, de modo que foi demonstrado a sua contribuição para esse estudo e para a ciência.

Neste estudo foram utilizados dados devidamente referenciados, identificando e respeitando seus autores, observando rigor ético quanto aos textos científicos pesquisados à propriedade intelectual e as demais fontes de pesquisas, no qual se diz respeito ao uso do conteúdo e de citações das obras consultadas.

\section{Resultados e Discussão}

A amostra final do estudo é composta de 15 publicações encontradas na base de dados LILACS; MEDLINE e SCIELO, que se encontram sumarizadas em duas tabelas a seguir. Na Tabela 1 é demonstrada a evolução anual dos artigos publicados no período de 2016 a 2021 em ordem cronológica, assim como as principais fontes de publicações relacionadas aos impactos psicológicos em pacientes pós-bariátricas. 
Tabela 1: Caracterização do estudo de acordo com autor, ano, título, base de dados, revista e local.

\begin{tabular}{|c|c|c|c|c|}
\hline $\mathbf{N}^{\mathbf{0}}$ & Autor e ano & Título & Base de Dados & Revista \\
\hline 01 & Távora et al., (2016) & $\begin{array}{l}\text { Gravidez pós-cirurgia bariátrica: perfil } \\
\text { psicossocial e assistência } \\
\text { Multiprofissional }\end{array}$ & MEDLINE & Revista do $\mathrm{CFCH}$ \\
\hline 02 & Paula et al., (2017) & $\begin{array}{l}\text { Avaliação Tipológica E Psicodinâmica } \\
\text { De Paciente Pós-Bariátrica }\end{array}$ & LILACS & Revista PsicoFAE \\
\hline $\mathbf{0 3}$ & $\begin{array}{l}\text { Santana; Oliveira; } \\
\text { Lacerda } \\
(2017)\end{array}$ & $\begin{array}{l}\text { Psicológico no Pré E Pós-Operatório De } \\
\text { Cirurgia Bariátrica }\end{array}$ & MEDLINE & Am J Psychiatry \\
\hline 04 & $\begin{array}{l}\text { Oliveira et al., } \\
(2018)\end{array}$ & $\begin{array}{l}\text { Repercussões da cirurgia bariátrica na } \\
\text { qualidade de vida de pacientes com } \\
\text { obesidade }\end{array}$ & SCIELO & RBONE \\
\hline 05 & $\begin{array}{l}\text { Alves; Viana } \\
(2018)\end{array}$ & $\begin{array}{l}\text { Qualidade de vida e risco de trnstorno } \\
\text { alimentar em pacientes submetidos a } \\
\text { cirúrgia bariatrica }\end{array}$ & LILACS & Rev. Ciênc. Méd \\
\hline 06 & $\begin{array}{l}\text { Monpellier et al., } \\
\text { (2018) }\end{array}$ & $\begin{array}{l}\text { Body image dissatisfaction and } \\
\text { depression in postbariatric patients is } \\
\text { associated with less weight loss and a } \\
\text { desire for body contouring surgery }\end{array}$ & MEDLINE & $\begin{array}{l}\text { Surg Obes Relat Dis } \\
\text {; }\end{array}$ \\
\hline 07 & $\begin{array}{l}\text { Marese; Tanaka; } \\
\text { Linartevichi } \\
\text { (2019) }\end{array}$ & $\begin{array}{l}\text { Interrelação entre cirurgia bariátrica e } \\
\text { transtorno depressivo } \\
\text { Maior }\end{array}$ & SCIELO & $\begin{array}{l}\text { Revista Thêma et } \\
\text { Scientia }\end{array}$ \\
\hline 08 & $\begin{array}{l}\text { Goes; Nascimento } \\
(2019)\end{array}$ & $\begin{array}{l}\text { Impactos psicológicos em pessoas } \\
\text { submetidas a cirurgia bariátrica }\end{array}$ & LILACS & Surg Obes Relat Dis \\
\hline 09 & $\begin{array}{l}\text { Stival et al., } \\
\text { (2019) }\end{array}$ & $\begin{array}{l}\text { Perspectiva da pessoa submetida à cirurgic } \\
\text { bariátrica }\end{array}$ & MEDLINE & $\begin{array}{l}\text { Revista Brasileira de } \\
\text { Obesidade, Nutrição } \\
\text { e Emagrecimento }\end{array}$ \\
\hline 10 & $\begin{array}{l}\text { Huang et al., } \\
(2019)\end{array}$ & $\begin{array}{l}\text { Predicted weight loss result of } \\
\text { laparoscopic sleeve gastrectomy: } \\
\text { Review of the first } 82 \text { consecutive } \\
\text { patients in an Asian bariatric unit. }\end{array}$ & MEDLINE & Asian J Surg \\
\hline 11 & $\begin{array}{l}\text { Arrudo; Jacinto; } \\
\text { Camargo } \\
(2020)\end{array}$ & $\begin{array}{l}\text { Vivências de pacientes submetidos à } \\
\text { cirurgia bariátrica sem } \\
\begin{array}{l}\text { Preparação prévia por equipe } \\
\text { multidisciplinar }\end{array}\end{array}$ & SCIELO & $\begin{array}{l}\text { Revista Enfermagem } \\
\text { em Evidência }\end{array}$ \\
\hline 12 & $\begin{array}{l}\text { Estevão et al., } \\
(2020)\end{array}$ & $\begin{array}{l}\text { Prevalência da probabilidade de } \\
\text { transtorno mental e fatores associados } \\
\text { entre indivíduos pós cirurgia bariátrica }\end{array}$ & LILACS & Cogitare enferm \\
\hline 13 & $\begin{array}{l}\text { Spirou; Raman; Smith } \\
(2020)\end{array}$ & $\begin{array}{l}\text { Psychological outcomes following } \\
\text { surgical and endoscopic bariatric } \\
\text { procedures: A systematic review. }\end{array}$ & MEDLINE & Obes Rev \\
\hline 14 & $\begin{array}{l}\text { Bringel Neto } \\
(2021)\end{array}$ & $\begin{array}{l}\text { Transtornos mentais em pacientes } \\
\text { submetidos à cirurgia } \\
\text { bariátrica: revisão integrativa de } \\
\text { literatura }\end{array}$ & LILACS & Surg Obes Relat Dis \\
\hline 15 & $\begin{array}{l}\text { Rodrigo; Faria } \\
(2021)\end{array}$ & $\begin{array}{l}\text { Os aspectos psicossociais da cirurgia } \\
\text { bariátrica: do pré ao pós-operatório }\end{array}$ & MEDLINE & $\begin{array}{l}\text { Cadernos } \\
\text { psicologia }\end{array}$ \\
\hline
\end{tabular}

Fonte: Pesquisa Direta (2021).

Para uma melhor compreensão do estudo, resultou-se à descrição destes, distribuindo os 15 artigos selecionados de acordo com os objetivos, resultados e conclusão, que serão analisados na Tabela 2.

Tabela 2: Distribuição dos artigos de acordo com os objetivos, resultados e conclusão.

\begin{tabular}{|c|c|c|c|c|}
\hline $\mathbf{N}^{\mathbf{0}}$ & $\begin{array}{l}\text { Autor } \\
\text { e ano }\end{array}$ & Objetivos & Resultados & Conclusão \\
\hline 01 & $\begin{array}{l}\text { Távora } \\
\text { et al., } \\
(2016)\end{array}$ & 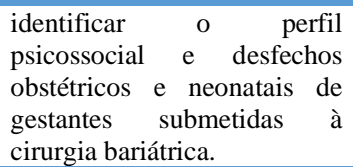 & $\begin{array}{l}\text { Salienta-se que as alterações de humor e ansiedade } \\
\text { observadas no estudo, com a tendência a desenvolver } \\
\text { depressão, ansiedade e compulsões no pós-operatório. }\end{array}$ & $\begin{array}{l}\text { A gravidez pós-cirurgia bariátrica é um } \\
\text { fenômeno complexo com aspectos múltiplos } \\
\text { que envolvem alterações fisiológicas, } \\
\text { questões psicológicas e exigências } \\
\text { nutricionais específicas. }\end{array}$ \\
\hline 02 & $\begin{array}{l}\text { Paula } \\
\text { et al., } \\
(2017)\end{array}$ & $\begin{array}{l}\text { Compreender a dinâmica } \\
\text { psíquica de uma paciente } \\
\text { pós-bariátrica e sua relação } \\
\text { com o comer }\end{array}$ & $\begin{array}{l}\text { A função sentimento está diretamente relacionada a } \\
\text { julgamentos de valor, ao relatar se os considera } \\
\text { saborosos ou não, sem considerar o efeito a longo prazo } \\
\text { de tal ação. Tal fato pode ter relação com as escolhas } \\
\text { alimentares e com a distorção que ela faz em relação à } \\
\text { quantidade e à qualidade dos alimentos com a } \\
\text { interferência de seu estado emocional. }\end{array}$ & $\begin{array}{l}\text { Foi possível compreender a existência entre } \\
\text { a relação do funcionamento psíquico da } \\
\text { paciente com o seu comportamento } \\
\text { alimentar. }\end{array}$ \\
\hline
\end{tabular}




\begin{tabular}{|c|c|c|}
\hline 03 & $\begin{array}{l}\text { Santan } \\
\text { a; } \\
\text { Oliveir } \\
\text { a; } \\
\text { Lacerd } \\
\text { a } \\
(2017)\end{array}$ & $\begin{array}{l}\text { Investigar aspectos } \\
\text { nutricionais e psicológico de } \\
\text { pacientes submetidos à } \\
\text { cirurgia bariátrica em uma } \\
\text { clínica particular de Aracaju- } \\
\text { SE. }\end{array}$ \\
\hline 04 & $\begin{array}{l}\text { Oliveir } \\
\text { a et al., } \\
\text { (2018) }\end{array}$ & 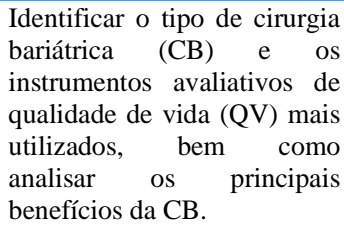 \\
\hline 05 & $\begin{array}{l}\text { Alves; } \\
\text { Viana } \\
\text { (2018) }\end{array}$ & $\begin{array}{l}\text { Avaliar o risco de transtorno } \\
\text { alimentar, bem como a } \\
\text { qualidade de vida no pós- } \\
\text { operatório de pacientes } \\
\text { submetidos a } \\
\text { bariátrica }\end{array}$ \\
\hline 06 & $\begin{array}{l}\text { Monpe } \\
\text { llier et } \\
\text { al., } \\
(2018)\end{array}$ & $\begin{array}{l}\text { Avaliar a relação entre } \\
\text { imagem corporal, sintomas } \\
\text { depressivos e perda de peso } \\
\text { em uma população pós- } \\
\text { bariátrica }\end{array}$ \\
\hline
\end{tabular}

07 Marese Discutir a influência do ; procedimento aplicado ao Tanaka tratamento da obesidade grau ; III no transtorno depressivo Linarte maior vichi (2019)

08 Goes; Avaliar os impactos A cirurgia bariátrica tem sido a intervenção mais Nascim psicológicos em pessoas utilizada para combater a obesidade, entretanto, pode ento submetidas a cirurgia bariátrica

09 Stival Descrever as percepções de et al., pessoas que foram (2019) submetidos à cirurgia bariátrica

10 Huang analisar os fatores que afetam et al., o resultado da perda de peso (2019) em nossos pacientes após bariátrica.

11 Arruda Conhecer as vivências de ; pacientes submetidos a Jacinto cirurgia bariátrica sem ; acompanhamento prévio por Camar equipe multidisciplinar go (2020)

12 Estevã Estimar a prevalência da o et al. probabilidade de transtorno (2020) mental comum e fatores associados entre indivíduos submetidos à cirurgia bariátrica

13 Spirou; Revisar sistematicamente os Raman resultados de depressão, ; Smith ansiedade e compulsão (2020) alimentar em diferentes momentos após a cirurgia bariátrica

14 Bringel Identificar variáveis

psicológicas descritas na literatura como associadas
aos pacientes que se
Dos pacientes analisados a maioria eram do gênero feminino, tinha mais de 2 anos de cirurgia, com idade entre 26 a 66 anos, antes da cirurgia a comorbidade mais prevalente foi a hipertensão arterial sistêmica (HAS), $64 \%$ e $52 \%$ fizeram o acompanhamento nutricional e psicológico pré-operatório e no pósoperatório $68 \%$ e $98 \%$ não compareceram para o acompanhamento respectivamente

Há uma melhora geral na QV percebida pelos pacientes no pós-operatório tardio.

$81,8 \%$ o sexo feminino teve prevalência na realização da cirurgia. Verificou-se uma associação entre a presença do transtorno alimentar e o tempo pós cirúrgico.

Nos pacientes que desejavam a cirurgia, a porcentagem de perda de peso total foi afetada negativamente pelos sintomas depressivos por meio da avaliação da aparência e da satisfação da área corporal.

Apesar da forte associação entre obesidade e depressão, não há consenso na relação de causa e efeito. Também não há dados precisos que correlacionem a depressão diretamente aos tipos de técnicas empregadas nenhuma delas evidenciou maior predisposição ao desenvolvimento e/ou manutenção ou melhora e/ou cura dos sintomas depressivos.

desencadear alguns transtornos alimentares que leva tanto ao excesso, quanto a perda de peso. Após a realização da cirurgia os mais comuns são: compulsão alimentar, bulimia e anorexia.

Além das condições complicadoras do pós-operatório, há também efeitos psicológicos negativos advindos da carga psíquica enquanto obesos.

Pacientes que relataram consumo de álcool no préoperatório, sem história psiquiátrica e sem osteoartrite apresentaram melhor tendência de atingir o peso ideal.

Os relatos das vivências emocionais destacamos dentre eles, que os maiores motivos para fazer a cirurgia foram: o excesso de peso, o preconceito social, dificuldade de locomoção

$50,2 \%$ apresentaram probabilidade de transtorno menta comum. Na análise múltipla, permaneceram associadas ao transtorno mental comum insônia

Os resultados sugeriram que a maioria dos pacientes experimenta uma redução de curto prazo nos sintomas de ansiedade e depressão desde o pré-cirurgia

Os estudos ainda são insuficientes para indicar que transtornos mentais em pacientes bariátricos. cirurgia bariátrica contribui para o desenvolvimento de
Apesar da melhora na qualidade de vida declarada pelos pacientes, é preciso que haja um estímulo maior para que os pacientes submetidos a cirurgias bariátricas mantenham o acompanhamento nutricional e psicológico no pós-operatório para prevenir possíveis complicações a médio ou longo prazo.

Conclui-se que uma das formas mais eficazes no tratamento da obesidade severa é a $\mathrm{CB}$, que faz com que os indivíduos reduzam o peso e tratem as comorbidades provenientes da obesidade, além da mudança de estilo de vida promovida pelos novos hábitos pós-cirúrgicos.

$\mathrm{O}$ desenvolvimento de transtornos alimentares nos pacientes pareceu depender do tempo pós cirúrgico e do grau de obesidade em que os pacientes se encontravam

antes da cirurgia.

Existem diferenças marcantes em relação à satisfação com a imagem corporal e aos sintomas depressivos ao comparar pacientes pós-bariátricos e sem bariátrica. A satisfação com a imagem corporal está associada a menos sintomas depressivos em todas as pacientes pós-bariátricas.

São necessários novos trabalhos que comparem as técnicas empregadas para o tratamento da obesidade mórbida e que relacionem as repercussões fisiológicas e psicopatológicas com as alterações anatômicas e funcionais decorrentes dos procedimentos.

Foi possível perceber que a cirurgia bariátrica imprime grande impacto sobre a esfera emocional do paciente. Diante do exposto, foi identificado que a $\mathrm{CB}$ não apresenta somente melhora na qualidade de vida, embora esteja notável as suas mudanças positivas.

As características individuais no processo de cirurgia bariatrica que as mudanças ocorreram para todos os entrevistados da pesquisa, em âmbito físico, psicológico e de relação social

Pacientes mais jovem, consumo de álcool pré-operatório e ausência de doença psiquiátrica foram fatores preditores positivos para perda de peso bem-sucedida após LSG.

Concluiu-se que os sujeitos têm consciência da importância da preparação prévia para amenizar ou eliminar complicações, favorecer maior adequação no processo de mudanças e adaptação à nova vida, à longo prazo

os resultados reforçam a importância da sistematização do cuidado nas ações de saúde em equipe multidisciplinar.

No geral, as complexas dificuldades psicológicas enfrentadas por indivíduos com obesidade continuam após a cirurgia e podem contribuir para a reincidência do peso a longo prazo.

A má absorção de nutrientes essenciais para o desenvolvimento orgânico contribui para tornar as pessoas suscetíveis ao desenvolvimento por reduzir os níveis de 
Research, Society and Development, v. 10, n. 17, e54101724081, 2021

(CC BY 4.0) | ISSN 2525-3409 | DOI: http://dx.doi.org/10.33448/rsd-v10i17.24081

\begin{tabular}{|c|c|c|c|c|}
\hline & & $\begin{array}{l}\text { submetem à cirurgia } \\
\text { bariátrica }\end{array}$ & & $\begin{array}{l}\text { ferro, vitamina B12 e serotonina como } \\
\text { elementos essenciais à saúde neurológica. }\end{array}$ \\
\hline 15 & $\begin{array}{l}\text { Rodrig } \\
\quad \text { o; } \\
\text { Faria } \\
(2021)\end{array}$ & $\begin{array}{l}\text { Compreender como o meio } \\
\text { social e familiar influenciam } \\
\text { em todo o processo de } \\
\text { tratamento cirúrgico }\end{array}$ & $\begin{array}{l}\text { Os resultados demonstram para a importância do } \\
\text { acompanhamento psicológico do período pré ao pós- } \\
\text { operatório, o que contribui para adaptação do indivíduo } \\
\text { ao novo corpo e ao novo estilo de vida }\end{array}$ & $\begin{array}{l}\text { Destaca-se a importância da Psicologia no } \\
\text { decorrer do preparo para a cirurgia até o seu } \\
\text { pósoperatório, buscando a melhor } \\
\text { compreensão do sujeito sobre suas angústias } \\
\text { já existentes e questões futuras advindas do } \\
\text { procedimento cirúrgico. }\end{array}$ \\
\hline
\end{tabular}

Fonte: Pesquisa Direta (2021).

Dentre os 15 artigos selecionados para o estudo, após a aplicação dos critérios de inclusão e exclusão, foram apresentados nas tabelas 1 e 2, as publicações selecionadas que tiver ame maior quantidade em publicações foram no ano de 2020, seguidas pelas contidas nos anos de 2019, 2021 e 2018. Tendo menor frequência de estudos selecionados o ano de 2016.

Na busca pelas informações à Base de Dados sete artigos foram do indexador da MEDLINE, dois da SCIELO e três da LILACS, em relação às informações e consultas em periódicos, observou-se que há uma variação entre os periódicos e locais de realização dos estudos. Houve um número considerável de publicações internacionais, o que reafirma ainda mais a importância do tema para os profissionais de Psicologia.

As tabelas 1 e 2 apresentadas anteriormente evidenciam uma amostra diversificada de trabalhos no que tange aos desfechos finais, a análise é positiva, uma vez que foi possível observar que há diversos impactos psicológicos em pacientes pós-bariátricos. Assim, diversos autores reforçaram a importância do acompanhamento seguro com profissionais qualificados da Psicologia e uma equipe multiprofissional pra melhorar a qualidade de vida.

Os artigos de modo geral tiveram o objetivo de descrever as perceções de pessoas que foram submetidos à cirurgia bariátrica, além de analisar os fatores que afetam o resultado da perda de peso em pacientes pós-bariátrica e avaliar os impactos psicológicos em pessoas submetidas a cirurgia bariátrica.

Em relação aos aspectos metodológicos houve uma diversidade, contudo, doze artigos foram pesquisa de campo e três pesquisa bibliográfica. As pesquisas de campos, caracterizaram-se qualitativa, por meio de entrevista semiestruturada com docentes do curso de psicologia e enfermagem em sete publicações, três foram relatos de caso do tipo exploratório.

Verificou-se que o sentimento está diretamente relacionado a julgamentos de valor, quando o assunto é alimentação, de modo que as pessoas comem por está ansiosa, depressiva, alegre, sem sentir fome e assim, o estado emocional pode contribuir para problemas emocionais, principalmente em pacientes obesos, que realizaram cirurgia bariatrica, como pode ser observado nos achados de Paula et al. (2017) e Távora et al. (2016).

Já os estudos realizados por Santana, Oliveira e Lacerda (2017), Monpellier et al. (2018), Spirou; Raman; Smith (2020) e Stival et al. (2019) analisaram a relação entre imagem corporal, sintomas depressivos e perda de peso em uma população pós-bariátrica e verificaram que inicialmente os pacientes queriam apenas a perda de peso total, contudo não estavam preparados psicologicamente para o pós-cirurgia e começam a ser afetados negativamente pela imagem construída e a real causando depressão, ansiedade, baixa autoestima, o que reforça a importância de profissionais de psicologia na equipe multiprofissional a tratar desses pacientes.

Ao analisar os achados de Stival et al. (2019) e Goes e Nascimento (2019) observa-se que a cirurgia bariátrica tem sido a intervenção mais utilizada para combater a obesidade, entretanto, essa intervenção médica contribui no pós-bariátrica para o surgimento de transtornos alimentares, reflexo da ansiedade, que pode influir na perda de peso e também no ganho após dois anos da cirurgia, os autores também recomendam o acompanhamento desses pacientes com psicólogos para reduzir essa carga emocional e apresentar um pós-cirurgia com qualidade de vida.

Para Arruda, Jacinto e Camargo (2020) as vivências emocionais após a cirurgia bariátrica sem acompanhamento prévio por equipe multidisciplinar são marcadas pelo excesso de peso, o preconceito social, a dificuldade de locomoção. 
Assim, há a consciência da importância da preparação prévia para amenizar ou eliminar complicações, para melhorar adequação no processo de mudanças à nova realidade, à longo prazo.

Estudos recentes realizados por Bringel Neto (2021) e Rodrigo; Faria (2021) identificaram variáveis psicológicas que se associam ao pós-operatório nas cirurgias bariátricas causando diversos transtornos devido a problemas de absorção de nutrientes essenciais para o desenvolvimento orgânico contribui para tornar as pessoas suscetíveis ao desenvolvimento por reduzir os níveis de ferro, vitamina B12 e serotonina como elementos essenciais à saúde neurológica, que contribui para o bem-estar, os estudos recomendam que a Psicologia esteja também no pós-operatório, buscando superar os problemas comuns que acometem os pacientes desse grupo clinico.

\section{Considerações Finais}

Esse estudo teve a contribuição em somar de maneira isenta e sem conflitos de interesses, de forma mais ampliada, no debate sobre o papel cada vez mais necessário do psicólogo no pós-operatório, em especial dos pacientes pós-bariátricos.

Vale destacar como os dados discutidos na discussão contribuem para a atuação do psicólogo organizacional, o que precisa ser melhorado e de sugestões para as próximas pesquisas. A relevância do psicólogo é atuar a partir de uma avaliação psicológica mais aprofundada, investigando o paciente e educando-o para as mudanças implicadas por meio da cirurgia. As indicações e contraindicações para as diversas cirurgias devem respeitar uma avaliação acurada dessa equipe multidisciplinar, da qual o psicólogo faz parte.

Vale reforçar que a ansiedade, independente da cirurgia, sempre se fez presente no dia a dia dos pacientes, porém, atualmente, é por muitas vezes considerada a sociedade da ansiedade, principalmente pela sobrecarga de prazos, pela competitividade na empresa e nos grupos sociais em geral, pela ditadura da magreza e dos corpos sarados, que impactam negativamente a vida das pessoas e precisam mudar esse padrão, de modo que esse estudo não se encerra aqui e pode ser abordado numa pós-graduação sobre a sociedade ansiosa e seus reflexos na vida social, profissional e financeira.

\section{Referências}

Alves, G. A., \& Viana, B. F. C. (2018). Qualidade de vida e risco de transtorno alimentar em pacientes submetidos a cirurgia bariátrica. Rev. Ciênc. Méd., 20(3), 87-98.

Arruda, J. C. (2019). Vivências de pacientes submetidos à cirurgia bariátrica sem preparação prévia por equipe multidisciplinar Revista Enfermagem em Evidência, 3(1), 92-102.

Bardal, A. G, Ceccatto, V., \& Mezzomo, T. R. (2016). Fatores de risco para recidiva de peso no pós-operatório tardio de cirurgia bariátrica. Scientia médica, 26(4), 10 .

Brasil. Ministério da Saúde (BR). (2020). Secretaria de Vigilância em Saúde. Secretaria de Gestão Estratégica e Participativa. Vigitel Brasil 2019: Vigilância de fatores de risco e proteção para doenças crônicas por inquérito telefônico. Brasília: Ministério da Saúde.

Bringel Neto, L. L. (2021). Transtornos Mentais em pacientes submetidos à cirurgia bariátrica: revisão integrativa de literatura. Surg Obes Relat Dis, 12(5), 45-67.

Cassin, S. et al. (2020). Food Addiction Is Associated with Binge Eating and Psychiatric Distress among Post-Operative Bariatric Surgery Patients and May Improve in Response to Cognitive Behavioural Therapy. Nutrients, 12(10), 2905.

Ercole, F. F, Melo, L. S, \& Alcoforado, C. L. G. C. (2014). Revisão integrativa versus revisão sistemática. Revista Mineira de Enfermagem, $18(1)$, 34-43.

Estevão, S. B et al. (2020). Prevalência da probabilidade de transtorno mental e fatores associados entre indivíduos pós cirurgia bariátrica. Cogitare enferm. 25(4), 90-109.

Ferreira, A. et al. (2016). O estudo de caso como método de pesquisa em enfermagem: uma revisão integrativa. Texto contexto - enferm. $26(4), 45-54$.

Huang, C. H et al. (2019). Predicted weight loss result of laparoscopic sleeve gastrectomy: Review of the first 82 consecutive patients in an Asian bariatric unit. Asian J Surg, 42(1), 373-378.

Macedo, C. O, Cardoso, R. F. A, \& Dias, D. A. M. (2019). Transtornos alimentares em pacientes pré e pós bariátricos: uma revisão da literatura. Revista Do Colégio Brasileiro de Cirurgiões, 45(60). 
Research, Society and Development, v. 10, n. 17, e54101724081, 2021

(CC BY 4.0) | ISSN 2525-3409 | DOI: http://dx.doi.org/10.33448/rsd-v10i17.24081

Marese, A. C, Tanaka, C, \& Linartevichi, V. F. (2019). Interrelação entre cirurgia bariátrica e transtorno depressivo maior. Revista Thêma et Scientia, 9(2).

Monpellier, V. M et al. (2018). Body image dissatisfaction and depression in postbariatric patients is associated with less weight loss and a desire for body contouring surgery. Surg Obes Relat Dis, 14(10), 1507-1515.

Moura, G. V, Sousa, M. C, \& Lima, C. H. R. (2021). Pós-Operatório De Cirurgia Bariátrica - Uma Revisão Integrativa Da Literatura. RECIMA - Revista Científica Multidisciplinar, 12(4), 4-18.

Oliveira, L. S. F et al. (2018). Repercussões da cirurgia bariátrica na qualidade de vida de pacientes com obesidade. RBONE - Revista Brasileira de Obesidade, Nutrição e Emagrecimento, 12(69), 47-58.

Paula, L. A et al. (2017). Avaliação tipológica e psicodinâmica de pacientepós-bariátrica. Pluralidades em Saúde Mental, 6(2), 85-96.

Rodrigues, J. O, \& Faria, H. M. (2019). Os aspectos psicossociais da cirurgia bariátrica: do pré ao pósoperatório. Cadernos De Psicologia, 2 (4), 551- 569.

Rosa, S. C et al. (2018). Perfil antropométrico e clínico de pacientes pós-bariátricos submetidos a procedimentos em cirurgia plástica. Rev. Col. Bras. Cir., 2, 45-57.

Santana, J. T, Oliveira, J. D, \& Lacerda, D. C. (2017). A importância do acompanhamento nutricional e psicológico no pré e pós-operatório de cirurgia bariátrica. Am J Psychiatry, 157(10), 1552-1562.

Spirou, D, Raman, J, \& Smith, E. (2020). Psychological outcomes following surgical and endoscopic bariatric procedures: A systematic review. Obes Ver. 21(6), 12-28.

Stival, N. L et al. (2019). Perspectiva da pessoa submetida à cirurgia bariátrica. Revista Brasileira de Obesidade, Nutrição e Emagrecimento, 13(77), 79-89.

Távora, G. F et al. (2016). Gravidez pós-cirurgia bariátrica: perfil psicossocial e assistência Multiprofissional. Revista do $C F C H$, $12(5)$, 1-6. 